\title{
Erratum: Chiral Effective Field Theory Predictions for Muon Capture on Deuteron and ${ }^{3} \mathrm{He}$ [Phys. Rev. Lett. 108, 052502 (2012)]
}

\author{
L. E. Marcucci, A. Kievsky, S. Rosati, R. Schiavilla, and M. Viviani
}

Q (Received 29 June 2018; published 25 July 2018)

DOI: 10.1103/PhysRevLett.121.049901

The relation between the low-energy constants (LECs) $c_{D}$ and $c_{E}$ in, respectively, the three-nucleon contact interaction and the two-nucleon contact axial current was given erroneously in Ref. [1]. The correct relation reads [2]

$$
d_{R}=-\frac{M_{N}}{4 \Lambda_{\chi} g_{A}} c_{D}+\frac{1}{3} M_{N}\left(c_{3}+2 c_{4}\right)+\frac{1}{6} .
$$

As a consequence of this error, the results of the Letter change as follows: (1) Figure 2 in the Letter should be replaced by Fig. 1 of this Erratum (note that Fig. 1 in the Letter showing the $c_{D}-c_{E}$ trajectories remains unchanged). (2) Tables I and II of the Letter are to be replaced by Tables I and II of this Erratum.

Differences between the results presented in Table II and the original table are generally very small, and therefore the conclusions of the Letter remain unchanged. The error above has also affected the studies of the proton-proton weak capture in Ref. [3] and muon capture in Ref. [4]. However, we expect the results and ensuing conclusions of those studies to remain unchanged as well since the LECs $c_{D}$ (or $d_{R}$ ) and $c_{E}$ are still constrained by fitting the same observables as in the Letter and, in particular, lead to the same enhancement as in the Letter needed to reproduce the tritium Gamow-Teller (GT) matrix element.

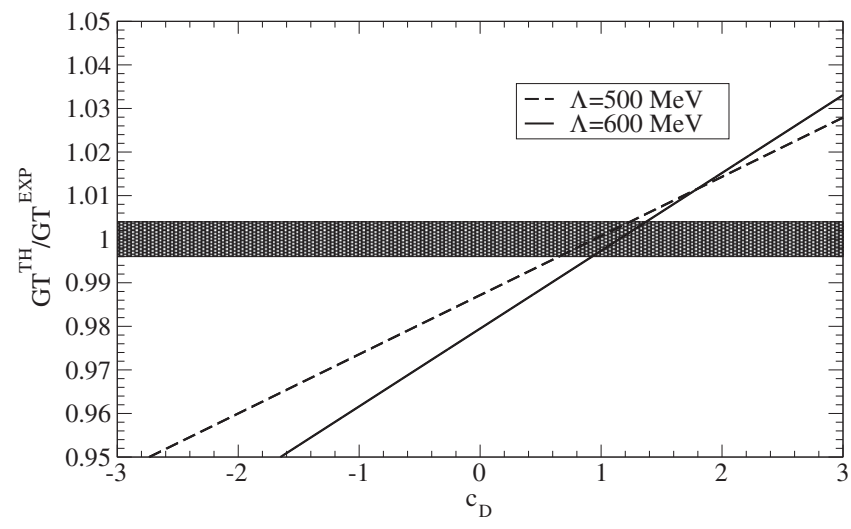

FIG. 1. The ratio $\mathrm{GT}^{\mathrm{TH}} / \mathrm{GT}^{\mathrm{EXP}}$ as a function of the $\mathrm{LEC} c_{D}$.

TABLE I. The LECs $g_{4 S}$ and $g_{4 V}$ associated with the isoscalar and isovector nucleon-nucleon contact terms in the electromagnetic current for $\Lambda=500$ and $600 \mathrm{MeV}$. See the Letter for further explanation.

\begin{tabular}{lccc}
\hline \hline & $\left\{c_{D}, c_{E}\right\}$ & $g_{4 S}$ & $g_{4 V}$ \\
\hline$\Lambda=500 \mathrm{MeV}$ & $\{1.24,0.001\}$ & $0.501 \pm 0.006$ & $0.883 \pm 0.003$ \\
& $\{0.65,-0.083\}$ & $0.500 \pm 0.005$ & $0.885 \pm 0.003$ \\
$\Lambda=600 \mathrm{MeV}$ & $\{1.37,-0.576\}$ & $0.447 \pm 0.006$ & $0.659 \pm 0.003$ \\
& $\{0.92,-0.643\}$ & $0.437 \pm 0.006$ & $0.644 \pm 0.003$ \\
\hline \hline
\end{tabular}


TABLE II. Total rates for muon capture on deuteron $\Gamma\left({ }^{2} \mathrm{H}\right)$ and ${ }^{3} \mathrm{He} \Gamma\left({ }^{3} \mathrm{He}\right)$, in $\mathrm{sec}^{-1}$, corresponding to $\Lambda=500$ and $600 \mathrm{MeV}$. The one-body (IA) and (one + two)-body (FULL) contributions are listed, along with the individual partial-wave contributions to $\Gamma\left({ }^{2} \mathrm{H}\right)$. Theoretical uncertainties in the FULL results, not reported when below $0.1 \mathrm{sec}^{-1}$, are due to the fitting procedure.

\begin{tabular}{lcccccccc}
\hline \hline & ${ }^{1} S_{0}$ & ${ }^{3} P_{0}$ & ${ }^{3} P_{1}$ & ${ }^{3} P_{2}$ & ${ }^{1} D_{2}$ & ${ }^{3} F_{2}$ & $\Gamma\left({ }^{2} \mathrm{H}\right)$ & \multicolumn{1}{c}{$\left.{ }^{3} \mathrm{He}\right)$} \\
\hline IA $(\Lambda=500 \mathrm{MeV})$ & 238.8 & 21.1 & 44.0 & 72.4 & 4.5 & 0.9 & 381.7 & 1355 \\
IA $(\Lambda=600 \mathrm{MeV})$ & 238.7 & 20.9 & 43.8 & 72.0 & 4.5 & 0.9 & 380.8 & 1351 \\
FULL $(\Lambda=500 \mathrm{MeV})$ & $253.7 \pm 0.9$ & 20.3 & 47.0 & 72.0 & 4.5 & 0.9 & $398.4 \pm 0.9$ & $1488 \pm 10$ \\
FULL $(\Lambda=600 \mathrm{MeV})$ & $253.3 \pm 1.0$ & 20.1 & 46.7 & 71.6 & 4.5 & 0.9 & $397.1 \pm 1.0$ & $1495 \pm 9$ \\
\hline \hline
\end{tabular}

[1] D. Gazit, S. Quaglioni, and P. Navrátil, Phys. Rev. Lett. 103, 102502 (2009).

[2] R. Schiavilla (unpublished).

[3] L. E. Marcucci, R. Schiavilla, and M. Viviani, Phys. Rev. Lett. 110, 192503 (2013).

[4] L. E. Marcucci and R. Machleidt, Phys. Rev. C 90, 054001 (2014). 